\title{
Exosomes with major histocompatibility complex class II and co-stimulatory molecules are present in human BAL fluid
}

\author{
C. Admyre*, J. Grunewald", J. Thyberg", S. Gripenbäck", G. Tornling ${ }^{\#}$, A. Eklund ${ }^{\#}$, A. Scheynius*, \\ S. Gabrielsson*
}

Exosomes with major histocompatibility complex class II and co-stimulatory molecules are present in human BAL fluid. C. Admyre, J. Grunewald, J. Thyberg, S. Gripenbäck, G. Tornling, A. Eklund, A. Scheynius, S. Gabrielsson. (C) ERS Journals Ltd 2003.

ABSTRACT: Exosomes are 30-100 nm diameter vesicles formed by inward budding of endosomal compartments and are produced by several cell types, including T-cells, Bcells and dendritic cells (DC)s. Exosomes from DCs express major histocompatibility complexes (MHC) class I and II, and co-stimulatory molecules on their surface, and can induce antigen-specific activation of $T$-cells.

The aims of the present study were to investigate for the presence of exosomes in bronchoalveolar lavage fluid (BALF) from healthy individuals, and to establish if these exosomes bear MHC and co-stimulatory molecules.

The authors analysed BALF taken from seven healthy volunteers and used exosomes from monocyte-derived DC (MDDC) cultures as a reference. After ultracentrifugation, exosomes were bound to anti-MHC class II coated magnetic beads and analysed by flow cytometry and electron microscopy.

The authors report for the first time that exosomes are present in BALF. These exosomes are similar to MDDC derived exosomes as they express MHC class I and II, CD54, CD63 and the co-stimulatory molecule CD86.

The results demonstrate that exosomes are present in the lung, and since they contain both major histocompatibility complex and co-stimulatory molecules it is likely that they are derived from antigen presenting cells and might have a regulatory role in local immune defence.

Eur Respir J 2003; 22: 578-583.
*Dept of Medicine, Unit of Clinical Allergy Research, "Division of Respiratory Medicine and Dept of Cell and Molecular Biology, Karolinska Hospital and Institutet, Stockholm, Sweden.

Correspondence: C. Admyre, Dept of Medicine, Unit of Clinical Allergy Research, Karolinska Hospital and Institutet, Stockholm, Sweden. Fax: 468335724

E-mail: Charlotte.Admyre@cfa.ki.se

Keywords: Bronchoalveolar lavage fluid fluid, CD54, CD86, exosomes, human leukocyte antigen-DR

Received: April 142003

Accepted after revision: May 292003

This work was supported by the Swedish Research Council (grants no. 16x-7924, 74x14182, and 6537), the Swedish Council for Work Life Sciences, the Swedish Asthma and Allergy Association's Research Foundation, the Hesselman Foundation, Konsul TH Bergs Foundation, the Swedish Heart-Lung Foundation, the Vårdal Foundation, the Centre for Allergy Research at Karolinska Institutet, the King Oscar II Jubilee Foundation, the King Gustaf V 80th Birthday Fund and the Karolinska Institutet.
Exosomes are $30-100 \mathrm{~nm}$ diameter vesicles produced by different cell types, including reticulocytes [1], platelets [2], Blymphocytes [3], T-lymphocytes [4], mast cells [5], epithelial cells [6] and dendritic cells (DC)s [7]. Exosomes are formed by inward budding of the limiting membrane of endosomal compartments, called multivesicular bodies (MVB)s [8]. In antigen presenting cells (APC)s MVBs are the main site for loading major histocompatibility complex (MHC) class II and recycling MHC class I molecules with exogenous antigens. The MHC molecules are located both on the limiting membrane and on the internal vesicles. When MVBs fuse with the plasma membrane the internal vesicles are released extracellularly and become exosomes [3]. APCs secrete exosomes that carry peptide-loaded MHC molecules, which can stimulate $\mathrm{T}$-cell proliferation in vitro [3]. T-cell stimulation has also been seen when using exosomes from rat mast cells engineered to express mouse or human MHC class II molecules [9]. In a mouse model, exosomes have shown a good potential for use as a tool in cancer therapies, where exosomes with tumour-peptide loaded MHC molecules were used for tumour eradication [7]. Exosomes from APCs do not only express $\mathrm{MHC}$ molecules, but also co-stimulatory molecules, such as CD86. They further express proteins not detected on the cell surface, such as the tetraspan proteins CD63 and CD82 [7], which interact with membrane proteins, such as integrins and human leukocyte antigen (HLA)-DR [10].

Exosomes may not only have a role in T-cell activation, but may also function as a communicator between cells in the immune system. Intestinal epithelial cells secrete exosome-like vesicles, which favour cross communication between the intestinal epithelium and the T-cells in the mucosa or in the blood [6]. Upon T-cell receptor (TCR) triggering, T-cells produce exosome-like vesicles expressing TCR and CD3, which may deliver signals to target cells baring the right MHC-peptide complex [4].

The lung is constantly exposed to inhaled particles and has numerous capillaries making its' mucosa a vulnerable surface between the environment and internal tissues. Therefore, it is important to have a powerful local immune defence. Whether exosomes are part of such a defence in the lung has not previously been addressed. The authors of the present study investigated if exosomes are present in human bronchoalveolar lavage fluid (BALF) from healthy individuals, using exosomes from monocyte-derived DCs (MDDCs) as a reference. The authors show for the first time, using flow cytometry and electron microscopy (EM), that exosomes are 
present in BALF. The authors found that these exosomes selected for MHC class II expression, such as exosomes derived from MDDC cultures, express HLA-DR, MHC class I, CD54, CD63 and the co-stimulatory molecule CD86.

\section{Materials and methods}

\section{Subjects}

BALF from seven healthy nonsmoking individuals aged 22-51 yrs (median 25 yrs), two males and five females, were analysed. None had a history of atopy, and all had normal serum Immunoglobulin (Ig)E values $\left(<120 \mathrm{kUL}^{-1}\right.$, ImmunoCap, Pharmacia Diagnostics, Uppsala, Sweden), normal chest radiographs and were without signs of respiratory infection for $\geqslant 1$ month prior to the study. The local ethics committee approved the study and informed consent was obtained from all subjects.

\section{Bronchoalveolar lavage fluid and handling of cells}

Bronchoscopy with bronchoalveolar lavage (BAL) (five aliquots of $50 \mathrm{~mL}$ phosphate buffered saline (PBS)) was performed as described previously [11]. The BALF was then strained through a double layer of Dacron nets (Millipore, Bedford, Ireland), and centrifuged at $400 \times g$ for $10 \mathrm{~min}$ at $4^{\circ} \mathrm{C}$. Cell viability was determined by Trypan blue exclusion and was $>90 \%$. For differential cell counts, cytospins were prepared at $20 \times g$ for $3 \mathrm{~min}$ and stained in May-Grünwald Giemsa. A total of 500 cells were counted.

\section{Generation of monocyte-derived dendritic cells from peripheral blood}

Buffy coats from six healthy blood donors at the Karolinska Hospital blood bank (Karolinska, Sweden) were used for generation of MDDCs as described previously [12, 13]. Peripheral blood mononuclear cells were isolated by density centrifugation on Ficoll Paque (Amersham Pharmacia Biotech AB, Uppsala, Sweden). CD14+ monocytes were positively selected using anti-CD14 microbeads (Miltenyi Biotech, Bergisch Gladbach, Germany) according to the manufacturer's instructions. Separated cells were analysed by flow cytometry for their expression of CD14 and purity ranged $88-96 \%$ (median $92 \%, n=6$ ). The cells were diluted to $4 \times 10^{5}$ cells $\cdot \mathrm{mL}^{-1}$ and cultured for 7 days in the presence of granulocyte/macrophage colony stimulating factor and interleukin 4 [13]. The medium was depleted from exosomes as described previously [14]. On day seven the MDDCs were analysed by flow cytometry and had a typical immature phenotype [13]. The expression of CDla ranged $81-91 \%$ (median 86\%, $\mathrm{n}=6$ ).

\section{Exosome preparations}

BALF stored at $-80^{\circ} \mathrm{C}$ from seven healthy volunteers or cell supernatants from MDDCs cultured for 7 days, were used for exosome preparations. The exosomes were purified as described previously with minor modifications [3]. The supernatants were centrifuged at $3,000 \times g$ for $20 \mathrm{~min}$ in room temperature (RT) and ultracentrifuged at $10,000 \times g$ for $30 \mathrm{~min}$ at $4^{\circ} \mathrm{C}$ to remove cell debris. For the collection of exosomes the supernatants were ultracentrifuged at 110,000 $\times g$ for $1 \mathrm{~h}$ at $4{ }^{\circ} \mathrm{C}$. The exosomes were washed and resuspended in PBS and stored at $-80^{\circ} \mathrm{C}$. Exosome samples were measured for protein content using BioRad DC protein assay (Bio-Rad Laboratories, Hercules, CA, USA) according to the manufacturer's instructions. The protein content of the exosome preparations from BALF ranged $0.20-0.51 \mu \mathrm{g}$ protein $\cdot \mathrm{mL}^{-1}$, median $0.30 \mu \mathrm{g}$ protein $\cdot \mathrm{mL}^{-1}$, of original volume $\mathrm{BALF}, \mathrm{n}=7$.

\section{Exosome coating to beads}

Exosomes were adsorbed onto dynabeads precoated with

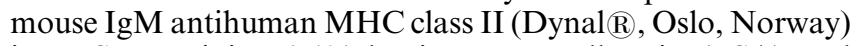
in PBS containing $0.1 \%$ bovine serum albumin (BSA) and $0.01 \%$ sodium-azide overnight at RT [15]. Pilot experiments with exosomes from MDDC supernatants (MDDC-exosomes) showed the beads to be saturated with exosomes at a concentration of $1 \mu \mathrm{g}$ protein to $1 \mu \mathrm{l}$ beads $\left(\sim 4 \times 10^{5}\right.$ beads). The authors also tested the saturation of beads with exosomes from BALF (BALF-exosomes). Here, the authors needed up to 10 times more protein to saturate the anti-MHC class II beads. Due to restricted amounts of BALF-exosomes the authors coated the beads with the amount of BALF-exosomes that gave a mean fluorescence intensity (MFI) of $\geqslant 17$ for HLA-DR. The MFI for the isotype matched-control antibody was normalised to 10 fluorescent units [15]. For EM the authors used 4-5 $\mu \mathrm{g}$ protein per $1 \mu \mathrm{L}$ beads for BALFexosomes and $1 \mu \mathrm{g}$ protein per $1 \mu \mathrm{L}$ beads for MDDCexosomes. Due to the limited amount of exosomes the authors could not analyse the exosomes from all individuals for all molecules with both flow cytometry and EM.

\section{Flow cytometry}

BALF-exosomes from seven healthy volunteers, MDDCexosomes and DCs from six blood donors were analysed by flow cytometry. The following mouse monoclonal antibodies (mAbs) were used: anti-HLA-DR (Fluorescein isothiocyanate (FITC)), anti-CD14 FITC and anti-CD54 phycoeythrin (PE) (Becton Dickinson, San Jose, CA, USA), anti-CD86 FITC, anti-CD80 FITC, anti-CD40 FITC, anti-MHC class I FITC, anti-CD63 PE (PharMingen, San Diego, CA, USA), and antiCD1a PE (Coulter Corporation, Hialeah, FL, USA) and compared with isotype-matched controls (Becton Dickinson). Cells or exosomes on beads were incubated with $\mathrm{mAbs}$ for $30 \mathrm{~min}$ on ice, in the dark, in PBS with $0.05 \%$ sodium-azide and $0.5 \%$ BSA for cells and in PBS with $0.01 \%$ sodium-azide and $0.1 \%$ BSA for exosomes. Samples were analysed by a Fluorescence-activated cell sorting calibur flow cytometer (Becton Dickinson). A minimum of $10^{4}$ cells and a minimum of $5 \times 10^{3}$ beads per sample were examined. The MFI for isotype-matched controls were normalised to 10 fluorescence units. The variation between FACS stainings was evaluated with MDDC-exosomes using pentaplicate determinations. The MFI for the isotype control antibody mouse $\mathrm{IgG}_{2 \mathrm{a}}-\mathrm{FITC}$ was $10.0 \pm 0.05,10 \pm 0.05$ for CD14 and $55.3 \pm 1.34$ for HLADR. Based on these observations the authors set the endpoint for a positive expression at $10.2(10.0+3 \times \mathrm{SD}$ for isotype control antibody).

Cells from BALF were analysed by flow cytometry for their expression of HLA-DR (DAKO Corporation, Carpinteria, CA, USA), CD14 (DAKO Corporation) and CD86 (Serotec, Oxford, UK) using primary antibodies and a secondary PEconjugated anti-mouse IgG antibody (DAKO Corporation). A minimum of $10^{4}$ cells per sample were examined in a FACSCalibur flow cytometer. 
Table 1.-Bronchoalveolar lavage fluid (BALF) recovery and cell count data from seven healthy individuals

BALF recovery $\%$

Total cell count ${ }^{\#} \times 10^{6}$

Total cell concentration ${ }^{\#} \times 10^{6} \mathrm{~L}^{-1}$

Macrophages ${ }^{\#} \%$

Lymphocytes ${ }^{\#} \%$

Neutrophils \# \%

Eosinophils $\%$

Basophils \# \%

Mast cells ${ }^{\top} \mathrm{n}$

HLA-DR ${ }^{+} \%$

CD14 $\%$

CD86 $6^{+} \%$
78 (67-84)

$16.1(10.4-22.3)$

$81.4(59.3-113.8)$

$94.0(88.4-96.5)$

$4.6(3.4-8.6)$

$0.6(0.4-3.0)$

$0.0(0.0-0.2)$

$0.0(0.0-0.2)$

3 (0-9)

$81(66-88)$

$41(20-58)$

$66(50-79)$
Data presented as median (range). HLA-DR: human leukocyte antigenDR; \#: cell count data from staining with May-Grünwald Giemsa; number of cells in 10 visual fields, $\times 16$ magnification; ${ }^{+}$: cell count data from flow cytometry analysis (minimum of $10^{4}$ cells per sample examined).

\section{Electron microscopy}

BALF-exosomes from three healthy volunteers and MDDCexosomes from one blood donor were coated to beads and incubated with a purified mouse $\mathrm{IgG}_{1}$ anti-CD63 $\mathrm{mAb}$ (PharMingen) and a purified rat $\mathrm{IgG}_{2 \mathrm{a}}$ anti-HLA-DR $\mathrm{mAb}$ (Accurate Chemical and Scientific Corporation, Westbury, NY, USA) overnight at $4{ }^{\circ} \mathrm{C}$. After repeated washings the beads were incubated with a $5 \mathrm{~nm}$ gold conjugated anti-rat IgG antibody and a $10 \mathrm{~nm}$ gold conjugated anti-mouse IgG antibody (Sigma Chemical Company). The exosomes-coated beads were then pelleted $(13,400 \mathrm{~g}, 30 \mathrm{~min}$, at RT) and fixed in $3 \%$ glutaraldehyde in $0.1 \mathrm{M}$ sodium cacodylate- $\mathrm{HCl}$ buffer ( $\mathrm{pH} 7.3$ ) with $0.05 \mathrm{M}$ sucrose. The specimens were postfixed in $1.5 \%$ osmium tetroxide in $0.1 \mathrm{M}$ cacodylate buffer $(\mathrm{pH} 7.3)$ with $0.5 \%$ potassium ferrocyanate for $90 \mathrm{~min}$ at $4{ }^{\circ} \mathrm{C}$, dehydrated in ethanol, stained with $2 \%$ uranyl acetate in ethanol, and embedded in epoxy resin. Sections of 50-70 nm thickness were cut with a Leica Ultracut (Leica/Reichert, Vienna, Austria), picked up on grids, stained with lead citrate, and examined in a Philips CM120 EM (Philips, Eindhoven, The Netherlands). The number of gold particles were counted on 50 exosomes from each preparation and compared with isotypematched controls, mouse $\mathrm{IgG}_{1}$ (Dako, Glostrup, Denmark) and rat $\operatorname{IgG}_{2 \mathrm{a}}$ (PharMingen), and the mean number of gold particles per exosome was calculated.

\section{Results}

\section{Bronchoalveolar lavage fluid cell counts}

BALF cells were retrieved and counted from all seven individuals (table 1). The results were similar to previous reports of BAL cells in healthy nonsmokers [16], with a majority of the cells characterised as macrophages, and a few as lymphocytes, mast cells or neutrophils. Occasionally, a few epithelial cells could be identified. BALF cells from all seven individuals were analysed by flow cytometry and found to express HLA-DR, CD14 and CD86 (table 1).

\section{Exosomes are present in bronchoalveolar lavage fluid}

Exosomes were detected in BALF from all seven individuals by flow cytometry. The BALF-exosomes expressed HLA-DR, CD54, CD63 and CD86 (table 2 and fig. 1). Further, exosomes from two out of three individuals showed expression of MHC class I (table 2). The molecules found on BALF-exosomes were in agreement with those detected on MDDC-exosomes (fig. 1). In addition, the MDDC-exosomes expressed CD40 and CD80, which were not detected on BALF exosomes. The level of MHC class I and II expression varied between BALF exosome donors, which was also seen for MDDC-exosomes (table 2). The authors could not detect any expression of CD3 or CD14 (table 2 and fig. 1).

Both BALF exosomes and MDDC exosomes had positive staining for CD63 and HLA-DR when analysed by immuno EM (fig. 2). The exosomes were $30-100 \mathrm{~nm}$ in size, in accordance with previous findings [17]. BALF-exosomes had a higher number of anti-CD63 labelling (median, 1.7; range, 1.2-2.1 antibodies/exosome) than anti-HLA-DR labelling (median, 0.1 ; range, $0.1-0.2$ antibodies/exosome). In contrast, MDDC exosomes had higher HLA-DR labelling (2.5 antibodies/ exosome) than CD63 labelling (0.6 antibodies/exosome). This was also evident when the exosomes were analysed by flow cytometry (table 2 and fig. 1).

\section{Discussion}

The authors showed for the first time that exosomes are present in BALF obtained from healthy individuals, and that they are similar to MDDC exosomes in that they express HLA-DR, MHC class I, CD63, as well as CD86 and CD54.

Table 2. - Characterisation of surface molecules on exosome-coated beads

\begin{tabular}{|c|c|c|c|c|}
\hline & \multicolumn{2}{|c|}{ BALF exosomes ${ }^{\#}$} & \multicolumn{2}{|c|}{ MDDC exosomes } \\
\hline & $\mathrm{MFI}^{+}$ & Experiments ${ }^{\top} \mathrm{n}$ & $\mathrm{MFI}^{+}$ & Experiments ${ }^{\top} \mathrm{n}$ \\
\hline HLA-DR & $20.6(17.0-33.4)$ & 7 & $63.6(49.3-117.3)$ & 6 \\
\hline MHC class I & $12.7(9.8-14.3)$ & 3 & $18.2(10.2-34.8)$ & 3 \\
\hline CD3 & $10.1(10.0-10.2)$ & 3 & $10.1(9.9-10.1)$ & 4 \\
\hline CD14 & $9.5(9.4-9.9)$ & 3 & $9.9(9.7-10.3)$ & 5 \\
\hline CD40 & $10.2(10.1-10.2)$ & 3 & $12.8(11.9-16.9)$ & 5 \\
\hline CD54 & $12.0(11.5-12.3)$ & 3 & $16.0(13.6-16.2)$ & 3 \\
\hline CD63 & $31.6(26.8-38.0)$ & 3 & $17.5(13.4-39.6)$ & 6 \\
\hline CD80 & $9.9,10.1$ & 2 & $10.8(10.5-12.4)$ & 5 \\
\hline CD86 & $12.1(11.7-13.0)$ & 3 & $18.7(15.1-21.3)$ & 5 \\
\hline
\end{tabular}

All data presented as median (range) unless otherwise stated. Values $>10.2$ were considered positive. BALF: bronchoalveolar lavage fluid; MFI: mean fluorescence intensity; MDDC: monocyte-derived dendritic cell; HLA-DR: human leukocyte antigen-DR; MHC: major histocompatibility complexes; ": exosomes from BALF taken from healthy individuals and exosomes from monocyte-derived dendritic cells cultured for 7 days from healthy blood donors were coated to anti-MHC class II magnetic beads and analysed by flow cytometric analysis for the expression of surface molecules. A minimum of $5 \times 10^{3}$ beads per sample were examined; ${ }^{\natural}$ : number of independent experiments using exosomes from different donors; ${ }^{+}:$the MFI for isotype-matched controls were normalised to 10 fluorescence units [15]. 

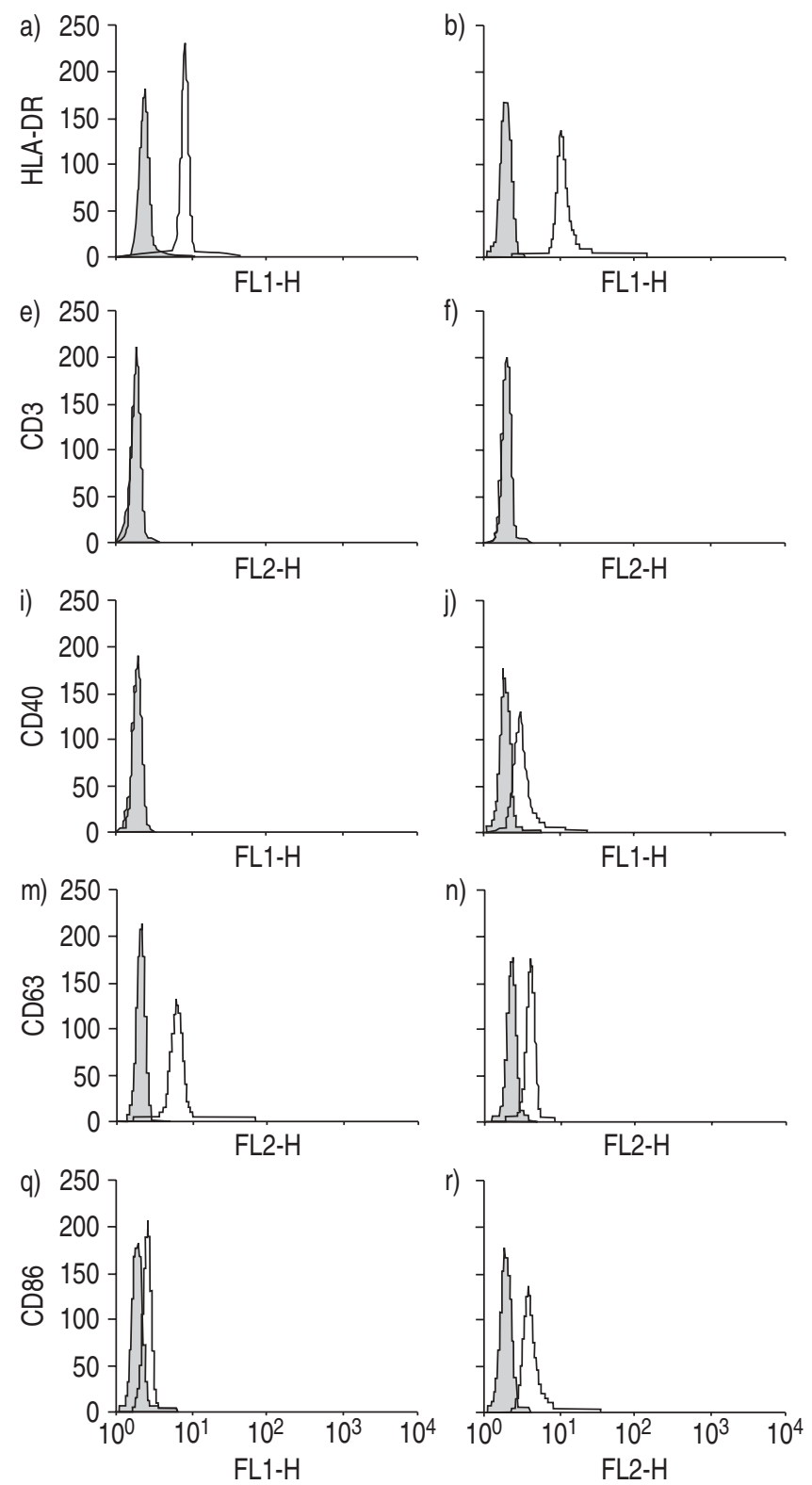

The protein levels needed to saturate the beads with MDDC exosomes were highly reproducible, supporting the theory that the MDDC supernatant is a reliable source of exosomes. Higher levels of protein were needed to saturate anti-MHC class II magnetic beads with BALF exosomes, probably because exosomes from BALF come from different cellular sources, including MHC class II negative cells. Another reason why higher protein levels are needed for saturation of anti-MHC class II magnetic beads with BALF exosomes compared with MDDC exosomes could be that the purification of exosomes from BALF might lead to co-purification of surfactant, which is a complex mixture of lipids and proteins of vital importance for lung function [18]. Therefore, surfactant could contribute to the total protein content in the exosome preparations from BALF. The reason that the MFI in general was lower for BALF exosomes compared with MDDC exosomes may be due to the beads that were not saturated due to the restricted amount of BALF exosomes.

The expression of MHC class I and II, CD63, CD86 and
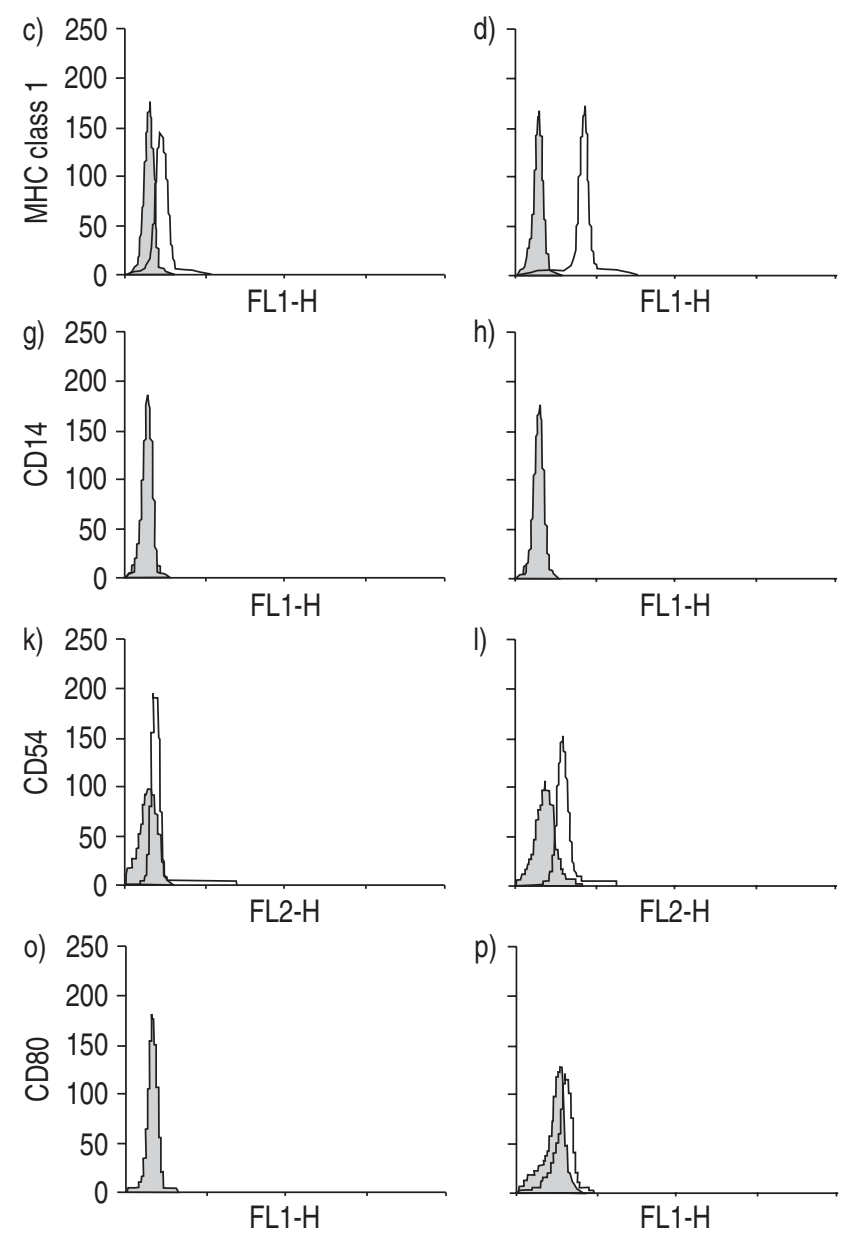

Fig. 1. - Expression of surface molecules exosomes coated beads, with exosomes from bronchoalveolar lavage fluid (a), c), e), g), i), k), m), o), q)) and monocyte derived dendritic cells supernatants (b), d), f), h), j), l), n), p), r)), with controls shown in. All exosomes were coated to antimajor histocompatibility complex (MHC) class II magnetic beads and analysed by flow cytometry. All antibodies used were compared with isotype-matched controls. A minimum of $5 \times 10^{3}$ beads per sample were examined. Results shown are from one experiment taken from the 2-7 experiments performed. HLA-DR: human leukocyte antigen-DR.

CD54 found on exosomes from DCs is in accordance with previous studies [7, 14, 15], as is also the absence of CD14 and the poor expression of CD80 [15]. However, the detection of CD40 on MDDC exosomes is contradictory to the findings of Clayton et al. [15] who were unable to detect CD40. MHC and co-stimulatory molecules are not only present on exosomes from DCs, therefore the exosomes detected in BALF might also be derived from other cell types. Exosomes from intestinal epithelial cells bear both MHC class I and II molecules, besides CD63 [6]. CD54, CD86, CD40 and MHC class II have been found on mast cell-derived exosomes [19]. Moreover, CD54, CD86, CD63 and MHC class I and II have been detected on exosomes derived from B-cells $[3,20,15]$. Exosomes from T-cells have been shown to bear CD63 and MHC class I and II molecules [4]. It would be expected that the majority of the exosomes detected in BALF originate from macrophages, since they were by far the most dominant cell type (table 1). Another possibility is that some of the exosomes are derived from cells that are not recovered in the BALF, e.g. epithelial cells. 

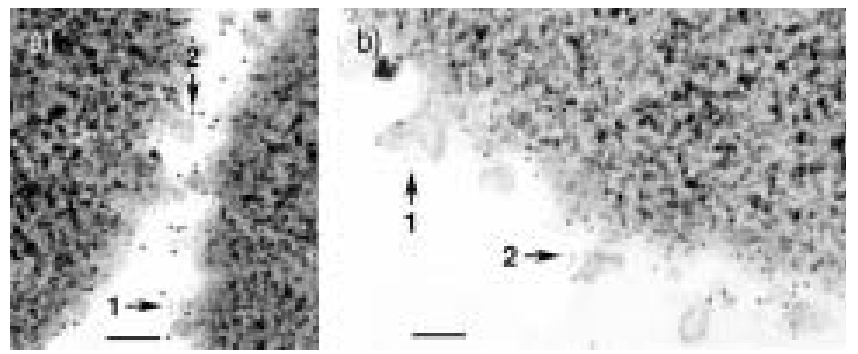

Fig. 2.-Exosomes detected by immune electron microscopy (EM). Exosomes from a) bronchoalveolar lavage and b) monocyte derived dendritic cells were coated to antimajor histocompatibility class II magnetic beads, stained for CD63 and human leukocyte antigen-DR HLA-DR using gold-conjugated antibodies and analysed by EM. The detection of HLA-DR (arrow 1) used $5 \mathrm{~nm}$ gold conjugated antibodies and $10 \mathrm{~nm}$ gold conjugated antibodies were used for the detection of CD63 (arrow 2). Scale bar=100 nm.

CD86 is a co-stimulatory molecule of great importance for prevention of anergy, induction of clonal expansion and differentiation of T-cells [21]. CD54 is an adhesion molecule that interacts with lymphocyte function-associated antigen (LFA)-1 to retain T-cells at the surface of APCs like DCs [22], and it can also deliver co-stimulatory signals to T-cells [23]. In addition, CD54 is important for cell migration. The fact that exosomes are located in the lung at the site of antigen entry and that these exosomes express MHC class II and I, as well as CD86 and CD54, is an indication that exosomes could be capable of homing to T-cell areas in lymph nodes and have a role in T-cell stimulation by adhering and presenting antigens, as well as providing co-stimulatory signals. In addition, they could have a role in stimulating memory $\mathrm{T}$-cells present in the lung. CD54 on exosomes could initiate adhesion to other cell types, thereby delivering signals between cells of the immune system. In a recent study, exosomes were found to be capable of promoting the exchange of functional peptide MHC complexes between DCs, which could increase the number of DCs bearing a certain peptide [24]. Another study indicated that follicular DCs can acquire microvesicles from other cells [25].

The exact function of exosomes in vivo is still unknown. APCs might secrete exosomes bearing antigen loaded MHC molecules, which could contribute to the stimulation of T-cells and give an amplified immune reaction. Alternatively, exosomes could be secreted to inhibit T-cell activation and keep the immune system in balance. Exosome-like structures, called tolerosomes, are released from small intestinal epithelial cells [26]. Tolerosomes carry MHC class II with bound antigenic peptide sampled from the gut lumen, and are capable of inducing antigen specific tolerance in naive recipient rats [26].

Taken together, the present study results demonstrate the presence of exosomes in bronchoalveolar lavage fluid. They exhibit surface presence of major histocompatibility complexes class I and II, as well as CD86, CD63 and CD54. This suggests that exosomes might have a role in antigen delivery or immune regulation during airway antigen exposure. The authors have preliminary data on the presence of exosomes expressing human leukocyte antigen -DR in bronchoalveolar lavage fluid from sarcoidosis patients and from birch pollen allergic patients during an allergic reaction. A future task will be to investigate the role of exosomes during inflammatory reactions in the lung.

\section{References}

1. Johnstone RM, Adams M, Hammond JR, Orr L, Turbide C. Vesicle formation during reticulocyte maturation. $J$ Biol Chem 1987; 262: 9412-9420.
2. Heijnen HFG, Schiel AE, Fijnheer R, Geuze HJ, Sixma JJ. Activated platelets release two types of membrane vesicles: microvesicles by surface shedding and exosomes derived from exocytosis of multivesicular bodies and alpha-granules. Blood 1999; 94: 3791-3799.

3. Raposo G, Nijman HW, Stoorvogel W, et al. B lymphocytes secrete antigen-presenting vesicles. J Exp Med 1996; 183: $1161-1172$

4. Blanchard N, Lankar D, Faure F, et al. TCR activation of human $\mathrm{T}$ cells induces the production of exosomes bearing the TCR/CD3/zeta complex. J Immunol 2002; 168: 3235-3241.

5. Raposo G, Tenza D, Mecheri S, Peronet R, Bonnerot C, Desaymard C. Accumulation of major histocompatibility complex class II molecules in mast cell secretory granules and their release upon degranulation. Mol Biol Cell 1997; 8: 2631-2645.

6. van Niel G, Raposo G, Candalh C, et al. Intestinal epithelial cells secrete exosome-like vesicles. Gastroenterology 2001; 121: 337-349.

7. Zitvogel L, Regnault A, Lozier A, et al. Eradication of established murine tumours using a novel cell-free vaccine: dendritic cell-derived exosomes. Nat Med 1998; 4: 594-600.

8. Pan B, Teng K, Wu C, Adam M, Johnstone RM. Electron microscopic evidence for externalization of the transferrin receptor in vesicular form in sheep reticulocytes. $J$ Cell Biol 1985; 101: 942-948.

9. Vincent-Schneider H, Stumptner-Cuvelette P, Lankar D, et al. Exosomes bearing HLA-DR1 molecules need dendritic cells to efficiently stimulate specific T-cells. Int Immunol 2002; 14: 713-722.

10. Rubinstein E, Le Naour F, Lagaudriere-Gesbert C, Billard M, Conjeaud H, Boucheix C. CD9, CD63, CD81, and CD82 are components of a surface tetraspan network connected to HLA-DR and VLA integrins. Eur J Immunol 1996; 26: 2657-2665.

11. Eklund A, Blaschke E. Relationship between changed alveolar-capillary permeability and angiotensin converting enzyme activity in serum in sarcoidosis. Thorax 1986; 41: 629-634.

12. Romani N, Gruner S, Brang D, et al. Proliferating dendritic cell progenitors in human blood. J Exp Med 1994; 180: 83-93.

13. Buentke E, Heffler LC, Wallin RPA, Löfman C, Ljunggren HG Scheynius A. The allergenic yeast Malassezia furfur induces maturation of human dendritic cells. Clin Exp Allergy 2001; 31: 1583-1593.

14. Théry C, Regnault A, Garin J, et al. Moleular characterization of dendritic cell-derived exosomes: selective accumulation of the heat shock protein hsc73. J Cell Mol Biol 1999; 147: 599 610 .

15. Clayton A, Court J, Navabi $\mathrm{H}$, et al. Analysis of antigen presenting cell derived exosomes, based on immunomagnetic isolation and flow cytometry. J Immunol Meth 2001; 247: 163-174.

16. Grunewald J, Eklund A, Katchar K, et al. Lung accumulations of eosinophil granulocytes after exposure to cornstarch glove powder. Eur Respir J 2003; 21: 646-651.

17. Théry C, Zitvogel L, Amigorena S. Exosomes: composition, biogenensis and function. Nat Rev Immunol 2002; 2: 569-579.

18. Creuwels LAJM, van Golde LMG, Haagsman HP. The pulmonary surfactant system: biochemical and clinical aspects. Lung 1997; 175: 1-39.

19. Skokos D, Le Panse S, Villa I, et al. Mast cell-dependant B and $\mathrm{T}$ lymphocyte activation is mediated by the secretion of immunologically active exosomes. J Immunol 2001; 166: 868876.

20. Escola JM, Kleijmeer MJ, Stoorvogel W, Griffith WJM, Yoshie O, Geuze HJ. Selective enrichment of tetraspan proteins on the internal vesicles of multivesicular endosomes and on exosomes secreted by human B-lymphocytes. $J$ Biol Chem 1998; 273: 20121-20127.

21. Koenen HJPM, Joosten I. Blockade of CD86 and CD40 induces alloantigen-specific immunoregulatory $\mathrm{T}$ cells that 
remain anergic even after reversal of hyporesponsiveness. Blood 2000; 95: 3153-3161.

22. van de Stolpe A, van der Saag PT. Intercellular adhesion molecule-1. J Mol Med 1996; 74: 13-33.

23. Chirathaworn C, Kohlmeier JE, Tibbetts SA, Rumsey LM, Chan MA, Benedict SH. Stimulation through intercellular adhesion molecule-1 provides a second signal for $\mathrm{T}$ cell activation. $J$ Immunol 2002; 168: 5530-5537.

24. Théry C, Duban L, Segura E, Véron P, Lantz O, Amigorena S.
Indirect activation of naive $\mathrm{CD}^{+} \mathrm{T}$ cells by dendritic cellderived exosomes. Nat Immunol 2002; 3: 1156-1162.

25. Denzer K, van Eijk M, Kleijmeer MJ, Jakobson E, de Groot C, Geuze HJ. Follicular dendritic cells carry MHC class IIexpressing microvesicles at their surface. J Immunol 2000; 165: 1259-1265.

26. Karlsson M, Lundin S, Dahlgren U, Kahu H, Pettersson I, Telemo E. "Tolerosomes" are produced by intestinal epithelial cells. Eur J Immunol 2001; 31: 2892-2900. 